\title{
Classification of integrable Vlasov-type equations
}

\author{
A.V. Odesskii ${ }^{1,2}$, M.V. Pavlov ${ }^{3}$, V.V. Sokolov ${ }^{2}$ \\ ${ }^{1}$ L.D. Landau Institute for Theoretical Physics (Russia) \\ ${ }^{2}$ University of Manchester (UK) \\ ${ }^{3}$ P.N. Lebedev Physical Institute (Russia)
}

\begin{abstract}
Classification of integrable Vlasov-type equations is reduced to a functional equation for a generating function. A general solution of this functional equation is found in terms of hypergeometric functions.
\end{abstract}

MSC numbers: 17B80, 17B63, 32L81, 14H70

Address: L.D. Landau Institute for Theoretical Physics of Russian Academy of Sciences, Kosygina 2, 119334, Moscow, Russia

E-mail: odesskii@itp.ac.ru, sokolov@itp.ac.ru 


\section{Introduction}

Consider the linear equation

$$
\Lambda_{t}=\{\Lambda, \psi\}
$$

where $\{\Lambda, \psi\}=\psi_{p} \Lambda_{x}-\psi_{x} \Lambda_{p}$. Here the unknown function $\Lambda(x, t, p)$ depends on the "spectral" parameter $p$ and a function $\psi=\psi(U(x, t), p)$. Following [1], we call (1.1) the Vlasov-type equation generated by $\psi(U, p)$. Equation (1.1) is also known as the dispersionless Lax equation.

It is easy to check that a partial hodograph transformation $\Lambda(x, t, p) \rightarrow p(x, t, \Lambda)$ reduces (1.1) to the following conservative form

$$
p_{t}=\psi(U, p)_{x}
$$

Here $\Lambda$ plays a role of parameter.

For some functions $\psi$ Vlasov-type equations are closely related to integrable hydrodynamic chains [2, 3]. A hydrodynamic chain associated with Vlasov-type equation can be derived by expanding $\Lambda(x, t, p)$ at a singular point of the function $\psi$. In such a case, formula (1.2) yields conservation laws for the hydrodynamic chain.

Example 1 (The Benney chain) [4, 5, 6, 1]. The Vlasov equation (or collisionless Boltzmann equation) has the form

$$
\Lambda_{t}+\Lambda_{p} U_{x}-p \Lambda_{x}=0
$$

where $\psi=\frac{p^{2}}{2}+U$. Substituting the expansion

$$
\Lambda=p+\frac{A^{0}}{p}+\frac{A^{1}}{p^{2}}+\frac{A^{2}}{p^{3}}+\frac{A^{3}}{p^{4}}+\ldots
$$

into (1.3), one derives the famous Benney hydrodynamic chain

$$
A_{t}^{k}=A_{x}^{k+1}+k A^{k-1} A_{x}^{0}, \quad k=0,1,2, \ldots
$$

where $A^{0}=U$. Let

$$
p=\Lambda-\frac{H_{0}}{\Lambda}-\frac{H_{1}}{\Lambda^{2}}-\frac{H_{2}}{\Lambda^{3}}-\frac{H_{3}}{\Lambda^{4}}-\ldots
$$

be the inverse series for (1.4). Functions $H_{i}$ can be easily calculated: $H_{0}=A^{0}, H_{1}=A^{1}, H_{2}=$ $A^{2}+\left(A^{0}\right)^{2}, \ldots$. The formula

$$
p_{t}=\left(\frac{p^{2}}{2}+U\right)_{x}
$$

generates infinitely many conservation laws for the Benney chain:

$$
\left(H_{k}\right)_{t}=\left(H_{k+1}-\frac{1}{2} \sum_{i=0}^{k-1} H_{i} H_{k-i-1}\right)_{x} .
$$


Example 2 (The Kupershmidt chain) [7, 8]. The Kupershmidt hydrodynamic chain

$$
B_{t}^{k}=B_{x}^{k+1}+B^{0} B_{x}^{k}+\beta k B^{k} B_{x}^{0}, \quad k=0,1,2, \ldots
$$

is connected to the Vlasov-type equation (1.1), where

$$
\psi=\frac{p^{\beta+1}}{\beta+1}+U p
$$

by the expansion

$$
\Lambda=p^{\beta}+B^{0}+\frac{B^{1}}{p^{\beta}}+\frac{B^{2}}{p^{2 \beta}}+\frac{B^{3}}{p^{3 \beta}}+\ldots,
$$

where $B^{0}=U$. Conservation laws for this chain can be calculated in the same way as in Example 1.

The hydrodynamic chains described in Examples 1, 2 admit infinitely many hydrodynamic reductions [9, 10]. The corresponding Vlasov-type equations admit the same reductions.

The following "integrable" functions $\psi$ were found in [2]:

Case 1. $\psi(U, p)=U+W(p)$, where

$$
W^{\prime \prime}=c_{1} W^{\prime 2}+c_{2} W^{\prime}+c_{3}
$$

and

Case 2. $\psi(U, p)=U W(p)$, where

$$
W^{\prime \prime}=\frac{1}{W}\left(c_{1} W^{\prime 2}+c_{2} W^{\prime}+c_{3}\right) .
$$

Here $c_{i}$ are arbitrary parameters. The Benney chain corresponds to Case 1 with $W(p)=p^{2} / 2$.

In this paper we describe all possible "integrable" functions $\psi(U, p)$ using the method of hydrodynamic reductions. The existence of hydrodynamic reductions have been proposed as a definition of integrability for dispersionless multi-dimensional equations in [9]. We apply this approach for Vlasov-type equations.

\section{Hydrodynamic reductions}

Suppose there exists a semi-Hamiltonian [11] hydrodynamic-type system

$$
r_{t}^{i}=v^{i}(\mathbf{r}) r_{x}^{i} \quad i=1,2, \ldots, N,
$$

and functions $U=u(\mathbf{r})$ and $\Lambda=\lambda(\mathbf{r}, p)$ such that these functions satisfy (1.1) for any solution $\mathbf{r}(x, t)$ of system (2.5). Then (2.5) is called a hydrodynamic reduction for the Vlasov-type equation (1.1). The partial hodograph transformation $\lambda(\mathbf{r}, p) \rightarrow p(\mathbf{r}, \lambda)$ leads to the corresponding hydrodynamic reduction of (1.2). 
Substituting $\Lambda=\lambda(\mathbf{r}, p)$ and $U=u(\mathbf{r})$ in (1.1) and (1.2), we obtain the equations

$$
\lambda_{t}=\psi_{p} \lambda_{x}-\psi_{x} \lambda_{p}
$$

and

$$
p_{t}=\psi(u, p)_{x} .
$$

Calculating the derivatives by virtue of (2.5), we obtain from (2.6) that

$$
\sum\left(\lambda_{p} \psi_{u} \partial_{i} u+\left[v^{i}(\mathbf{r})-\psi_{p}\right] \partial_{i} \lambda\right) r_{x}^{i}=0,
$$

where we use the notation $\partial_{i}=\partial / \partial r^{i}$. Since $\mathbf{r}$ is arbitrary solution of system (2.5), we get

$$
\lambda_{p} \psi_{u} \partial_{i} u=\left[\psi_{p}-v^{i}(\mathbf{r})\right] \partial_{i} \lambda, \quad i=1,2, \ldots, N .
$$

Let us determine functions $p^{i}(\mathbf{r}), i=1,2, \ldots, N$ as solutions of the equations

$$
v^{i}(\mathbf{r})=\left.\psi_{p}\right|_{p=p^{i}} .
$$

Then (2.8) implies that the equation $\lambda_{p}=0$ has $N$ solutions (pairwise distinct in the generic case), i.e.

$$
\left.\lambda_{p}\right|_{p=p^{i}}=0, \quad i=1,2, \ldots, N .
$$

Without loss of generality we can fix the Riemann invariants $r^{i}$ of the system (2.5) in such a way that

$$
r^{i}=\left.\lambda\right|_{p=p^{i}} .
$$

Indeed, if we substitute $p=p^{i}$ into equation (2.6), then (2.6), (2.10) imply

$$
\left(\left.\lambda\right|_{p=p^{i}}\right)_{t}=\left(\left.\psi_{p}\right|_{p=p^{i}}\right)\left(\left.\lambda\right|_{p=p^{i}}\right)_{x} .
$$

This means (see (2.9)) that $\left.\lambda\right|_{p=p^{i}}$ satisfies (2.5) and therefore $\left.\lambda\right|_{p=p^{i}}=R_{i}\left(r^{i}\right)$ for some functions $R_{i}$. According to (2.10), the branch points of the Riemann surface determined by the equation $\Lambda=\lambda(\mathbf{r}, p)$, are nothing but the Riemann invariants of system (2.5). This fact is well-known for hydrodynamic-type systems that produced by the Whitham averaging procedure applied to multi-phase solutions of both integrable continuous dispersive equations and integrable discrete equations (see references in [3]).

Substituting functions $p(\mathbf{r}, \lambda), u(\mathbf{r})$ in (2.7), we obtain

$$
\partial_{i} p=\frac{\psi_{u} \partial_{i} u}{\left.\psi_{p}\right|_{p=p^{i}}-\psi_{p}} .
$$

If we fix $\lambda=r^{k}, k \neq i$, then (2.11) implies $p=p^{k}$ and we obtain

$$
\partial_{i} p^{k}=\frac{\left.\psi_{u}\right|_{p=p^{k}} \partial_{i} u}{\left.\psi_{p}\right|_{p=p^{i}}-\left.\psi_{p}\right|_{p=p^{k}}} .
$$


Let us introduce the following notation:

$$
f_{i}=\frac{\psi_{u}}{\left.\psi_{p}\right|_{p=p^{i}}-\psi_{p}}, \quad f_{i k}=\frac{\left.\psi_{u}\right|_{p=p^{k}}}{\left.\psi_{p}\right|_{p=p^{i}}-\left.\psi_{p}\right|_{p=p^{k}}}, \quad i \neq k .
$$

The compatibility conditions $\partial_{k}\left(\partial_{i} p\right)=\partial_{i}\left(\partial_{k} p\right), \quad i \neq k$ are equivalent to the equations

$$
\partial_{i k}^{2} u=\frac{f_{i k} \partial_{p^{k}} f_{k}-f_{k i} \partial_{p^{i}} f_{i}+\partial_{u}\left(f_{k}-f_{i}\right)+f_{i} \partial_{p} f_{k}-f_{k} \partial_{p} f_{i}}{f_{i}-f_{k}} \partial_{i} u \partial_{k} u .
$$

Equations (2.13), (2.14) form a system of equations named in 2 the generalized Gibbons-Tsarev system (cf. [10]).

Since $u$ does not depend on $p$, (2.14) implies the following functional equation

$$
\partial_{p}\left(\frac{f_{i k} \partial_{p^{k}} f_{k}-f_{k i} \partial_{p^{i}} f_{i}+\partial_{u}\left(f_{k}-f_{i}\right)+f_{i} \partial_{p} f_{k}-f_{k} \partial_{p} f_{i}}{f_{i}-f_{k}}\right)=0
$$

for the function $\psi(u, p)$. In the next sections we study this functional equation and found its general solution. The general solution is expressed in terms of a pair of arbitrary solutions of the standard hypergeometric equation

$$
u(u-1) y(u)^{\prime \prime}+[(\alpha+\beta+1) u-\gamma] y(u)^{\prime}+\alpha \beta y(u)=0 .
$$

Note that the general solution of the Chazy equation, which appears in the classification paper [12], can also be parameterized by a pair of hypergeometric functions. Our solution $\psi(u, p)$ is a generalization of the solution $h(\xi, u)$ found in [13] (see Example 4).

\section{Particular solutions}

In Sections 4, 5 we solve the functional equation (2.15) in terms of quadratures of hypergeometric functions. In this section we consider some particular cases, where the result can be written more explicitly.

Computing the numerator of the left hand side of (2.15) and expanding it at $p^{i}=p, p^{k}=p$, we obtain that the vanishing of the coefficients up to 8-th degree in the corresponding Taylor series is equivalent to the following system of PDEs for the function $\psi(u, p)$ :

$$
\begin{aligned}
& 3 \psi_{p p p}^{3} \psi_{u}^{3}-4 \psi_{p p} \psi_{p p p} \psi_{p p p p} \psi_{u}^{3}+\psi_{p p}^{2} \psi_{p p p p p} \psi_{u}^{3}-3 \psi_{p p} \psi_{p p p}^{2} \psi_{u}^{2} \psi_{p u}+2 \psi_{p p}^{2} \psi_{p p p p} \psi_{u}^{2} \psi_{p u}+ \\
& 6 \psi_{p p}^{2} \psi_{p p p} \psi_{u}^{2} \psi_{p p u}-5 \psi_{p p}^{3} \psi_{u}^{2} \psi_{p p p u}-6 \psi_{p p}^{4} \psi_{p u} \psi_{u u}+6 \psi_{p p}^{4} \psi_{u} \psi_{p u u}=0 \\
& 3 \psi_{p p p}^{2} \psi_{u}^{3} \psi_{p p u}-\psi_{p p} \psi_{p p p p} \psi_{u}^{3} \psi_{p p u}-3 \psi_{p p} \psi_{p p p} \psi_{u}^{3} \psi_{p p p u}+\psi_{p p}^{2} \psi_{u}^{3} \psi_{p p p p u}-3 \psi_{p p}^{2} \psi_{p p p} \psi_{u} \psi_{p u} \psi_{u u}- \\
& 6 \psi_{p p}^{3} \psi_{p u}^{2} \psi_{u u}+3 \psi_{p p}^{3} \psi_{u} \psi_{p p u} \psi_{u u}+3 \psi_{p p}^{2} \psi_{p p p} \psi_{u}^{2} \psi_{p u u}+6 \psi_{p p}^{3} \psi_{u} \psi_{p u} \psi_{p u u}-3 \psi_{p p}^{3} \psi_{u}^{2} \psi_{p p u u}=0
\end{aligned}
$$


and

$$
\begin{aligned}
& -3 \psi_{p p p}^{2} \psi_{u}^{2} \psi_{p u} \psi_{u u}+\psi_{p p} \psi_{p p p p} \psi_{u}^{2} \psi_{p u} \psi_{u u}-6 \psi_{p p} \psi_{p p p} \psi_{u} \psi_{p u}^{2} \psi_{u u}-6 \psi_{p p}^{2} \psi_{p u}^{3} \psi_{u u}+ \\
& 3 \psi_{p p} \psi_{p p p} \psi_{u}^{2} \psi_{p p u} \psi_{u u}+6 \psi_{p p}^{2} \psi_{u} \psi_{p u} \psi_{p p u} \psi_{u u}-\psi_{p p}^{2} \psi_{u}^{2} \psi_{p p p u} \psi_{u u}+3 \psi_{p p p}^{2} \psi_{u}^{3} \psi_{p u u}- \\
& \psi_{p p} \psi_{p p p p} \psi_{u}^{3} \psi_{p u u}+6 \psi_{p p} \psi_{p p p} \psi_{u}^{2} \psi_{p u} \psi_{p u u}+6 \psi_{p p}^{2} \psi_{u} \psi_{p u}^{2} \psi_{p u u}-3 \psi_{p p}^{2} \psi_{u}^{2} \psi_{p p u} \psi_{p u u}- \\
& 3 \psi_{p p} \psi_{p p p} \psi_{u}^{3} \psi_{p p u u}-3 \psi_{p p}^{2} \psi_{u}^{2} \psi_{p u} \psi_{p p u u}+\psi_{p p}^{2} \psi_{u}^{3} \psi_{p p p u u}=0
\end{aligned}
$$

This system and the functional equation (2.15) are invariant under any transformation of the form $u \rightarrow f(u)$. They are also invariant with respect to the following symmetry group:

$$
\psi \rightarrow c_{2} \psi+c_{1} p+c_{0}, \quad p \rightarrow k_{2} \psi+k_{1} p+k_{0}
$$

These symmetries are associated with linear transformations of independent variables in (1.1), (1.2). Notice that any function $\psi=W(u)+p V(u)$ satisfies equation (2.15) and system (3.17) (3.19).

The integrable cases $\psi(u, p)=u+W(p)$ and $\psi(u, p)=u W(p)$ described in Introduction can be found directly from (3.17). We denote these cases as Case 1 and Case 2, respectively. We mention two more particular integrable cases:

Case 3. $\psi(u, p)=W(u-p)$, where

$$
W^{\prime \prime}=c_{1} W^{\prime 3}+c_{2} W^{\prime 2}+c_{3} W^{\prime}
$$

Case 4. $\psi(u, p)=p u+W(p)$, where

$$
W^{\prime \prime}=\frac{1}{p}\left(c_{1} W^{\prime 2}+c_{2} W^{\prime}+c_{3}\right) .
$$

Here $c_{i}$ are arbitrary constants. The Kuperhsmidt chain belongs to Case 4 . It is easy to see that Case 3 is connected to Case 1 by the transformation $\psi \leftrightarrow p$. In all these four particular cases system (3.17)-(3.19) is equivalent to some ordinary differential equation of the fifth order for the function $W$.

System (3.17)-(3.19) admits the substitution

$$
\psi_{p}=F(p, \psi)
$$

corresponding to the factorization with respect to the symmetry group $u \rightarrow f(u)$. As the result, one gets an overdetermined system of three PDEs for the function $F(x, y)$. We do not present this system here because of its complexity.

Case $\mathbf{1}$ corresponds to the case $\overline{\mathbf{1}}: F(x, y)=V(x)$, where $V=W^{\prime}$. Case $\mathbf{3}$ corresponds to $\overline{3}: F(x, y)=V(y)$. Case 2 corresponds to $\overline{2}: F(x, y)=y V(x)$, where $V=W^{\prime} / W$. Case 4 corresponds to $\overline{4}: F(x, y)=y / x+V(x)$, where $V=W^{\prime}-W / x$. 
Another particular solution is determined by the function

$$
F(x, y)=\frac{2 x+\alpha}{x^{2}+\alpha x+\beta} \cdot \frac{y^{2}+\gamma y+\delta}{2 y+\gamma} .
$$

The corresponding equation (3.21) leads to equation (2.7) of the form

$$
p_{t}=\partial_{x} \sqrt{u\left(p^{2}+t_{1}\right)-t_{2}}
$$

where $t_{i}$ are arbitrary constants. In this example the function $F$ has the form

$$
F(x, y)=\frac{V(y)}{W(x)}
$$

An investigation of this ansatz leads to the following result. The case, where $V$ or $W$ is a linear function, was considered above. Namely, the cases $W=1, V=1$ and $V=y$ coincide with Cases 3, 1 and 2, respectively. The case $W=x$ transforms to Case 2 by the substitution $\psi \leftrightarrow p$. If $V^{\prime \prime} \neq 0$ and $W^{\prime \prime} \neq 0$, then the following three classes of solutions (3.23) exist:

Case 5. $\quad V V^{\prime \prime}=2 V^{\prime^{2}}+c_{1} V^{\prime}+c_{2}, \quad W W^{\prime \prime} 2 W^{\prime^{2}}+c_{1} W^{\prime}+c_{2}$,

Case 6. $\quad V V^{\prime \prime}={V^{\prime 2}}^{2}+c_{1} V^{\prime}+c_{2}, \quad W W^{\prime \prime}=W^{\prime^{2}}+c_{1} W^{\prime}+c_{2}$,

Case 7. $\quad V V^{\prime \prime}=-{V^{\prime 2}}^{2}+c_{1} V^{\prime}+c_{2}, \quad W W^{\prime \prime}=-W^{\prime^{2}}+c_{1} W^{\prime}+c_{2}$.

For all these cases the generic solution depends on 6 arbitrary parameters. Function (3.22) belongs to Case 5 with $c_{1}=-3, c_{2}=1$.

\section{General solution}

Let us expand the left hand side of (2.15) into the Taylor series at $p^{k}=p$. Denote by $S\left(p^{i}, p\right)$ the first nontrivial coefficient of this expansion. For fixed $p$ consider $S=0, S_{p}=0$ as a system of linear algebraic equations with respect to derivatives $\psi_{u}, \psi_{u p^{i}}$. Its determinant does not vanish if $\psi_{u} \psi_{p p} \neq 0$. Solving this system, we obtain

$$
\psi_{u}=\frac{Q\left(\psi_{p}\right)}{\psi_{p p}},
$$

where $Q$ is a polynomial with respect to $\psi_{p}$ of degree not greater than 4 with coefficients depending on $u$ only. Taking into account (4.24), it is easy to extract from the equation $S_{p^{i}}=0$ that

$$
\frac{\psi_{p p p}}{\psi_{p p}^{2}}=\frac{R\left(\psi_{p}\right)}{Q\left(\psi_{p}\right)}
$$

where $R$ is a polynomial of degree not greater than three. The compatibility condition of equations (4.24) and (4.25) has the form

$$
Q^{2} Q_{x x x}-R Q Q_{x x}+R Q_{x}^{2}-\left(R^{2}+R_{x} Q\right) Q_{x}+R Q_{u}-R_{x x} Q^{2}+\left(2 R R_{x}-R_{u}\right) Q=0
$$


where $x=\psi_{p}$.

Assume that the polynomial

$$
Q(x)=a\left(x-b_{1}\right)\left(x-b_{2}\right)\left(x-b_{3}\right)\left(x-b_{4}\right)
$$

has distinct roots and rewrite (4.25) as

$$
\frac{\psi_{p p p}}{\psi_{p p}^{2}}=\frac{k_{1}}{\psi_{p}-b_{1}}+\ldots+\frac{k_{4}}{\psi_{p}-b_{4}} .
$$

One can verify that the system consisting of equations (3.17)-(3.19) and (4.26) is equivalent to the following :

1. The functions $k_{i}(u)$ are arbitrary constants such that $k_{1}+\ldots+k_{4}=3$.

2. The functions $a(u), b_{i}(u)$ satisfy the following system of ODEs:

$$
b_{i}^{\prime}=a\left(1-k_{i}\right) \prod_{j \neq i}\left(b_{i}-b_{j}\right), \quad i=1, \ldots, 4 .
$$

The function $a(u)$ can be chosen arbitrarily due to the admissible transformations $u \rightarrow s(u)$. Consider the double ratio

$$
\rho=\frac{\left(b_{1}-b_{2}\right)\left(b_{3}-b_{4}\right)}{\left(b_{1}-b_{3}\right)\left(b_{2}-b_{4}\right)} .
$$

Differentiating $\rho$ by virtue of (4.28), it is easy to check that $\rho^{\prime} \neq 0$. Let us change $u$ in such a way that $\rho=u$. This means that we choose

$$
a=\frac{1}{\left(b_{2}-b_{3}\right)\left(b_{1}-b_{4}\right)}+\frac{1}{\left(b_{1}-b_{2}\right)\left(b_{3}-b_{4}\right)} .
$$

One can verify that the formulae

$$
b_{1}=\frac{z_{2}+u y_{2}}{z_{1}+u y_{1}}, \quad b_{2}=\frac{y_{2}}{y_{1}}, \quad b_{3}=\frac{z_{2}+y_{2}}{z_{1}+y_{1}}, \quad b_{4}=\frac{z_{2}}{z_{1}}
$$

where $\left(y_{1}, z_{1}\right),\left(y_{2}, z_{2}\right)$ are two arbitrary solutions of the linear system

$$
y^{\prime}=\frac{k_{1}+k_{2}+k_{3}-2}{u-1} y+\frac{k_{1}+k_{2}+k_{3}-2}{u(u-1)} z, \quad z^{\prime}=\frac{1-k_{1}}{u-1} y+\frac{1-k_{1}-k_{2}+k_{2} u}{u(u-1)} z,
$$

define a general solution of (4.28). Notice that if $k_{1}+k_{2}+k_{3} \neq 2$, then

$$
z=-u y+\frac{u(u-1)}{k_{1}+k_{2}+k_{3}-2} y^{\prime}
$$

and system (4.29) is equivalent to the hypergeometric equation (2.16), where $k_{1}=1+\alpha-\gamma$, $k_{2}=1-\alpha, k_{3}=\gamma-\beta$. 
System (4.24), (4.25) can be reduced to quadratures by the following way. Let us determine a function $\phi(u, p)$ as the solution of the over-determined system:

$$
\phi_{u}=-\frac{\phi(\phi-1) y_{1}^{\prime}}{\beta\left(y_{1} \phi+z_{1}\right)}, \quad \phi_{p}=\frac{\phi^{k_{1}}(\phi-u)^{k_{2}}(\phi-1)^{k_{3}}}{y_{1} \phi+z_{1}} .
$$

It is easy to check that this system is consistent. Then the solution of the following system in involution

$$
\psi_{u}=\frac{y_{2} y_{1}^{\prime}-y_{1} y_{2}^{\prime}}{\beta\left(y_{1} \phi+z_{1}\right)} \phi^{1-k_{1}}(\phi-u)^{1-k_{2}}(\phi-1)^{1-k_{3}}, \quad \psi_{p}=\frac{y_{2} \phi+z_{2}}{y_{1} \phi+z_{1}}
$$

is a general solution of (2.15). This fact can be verified by a direct calculation. It turns out that the expression under differentiating in the left hand side of (2.15) is equal to

$$
\frac{(1-u-\beta) \alpha^{2}+(1-u-\alpha) \beta^{2}+u(4 \alpha \beta-\alpha-\beta)}{u(u-1)(\alpha-\beta)^{2}},
$$

where $\alpha=\phi\left(u, p^{i}\right), \beta=\phi\left(u, p^{k}\right)$.

Remark. The standard Wronskian formula for second order linear ODE implies that the expression $y_{2} y_{1}^{\prime}-y_{1} y_{2}^{\prime}$ from (4.31) equals $C u^{k_{1}+k_{2}-2}(u-1)^{k_{2}+k_{3}-2}$ for some constant $C$.

\section{Degenerations}

In Section 4 we have considered the general case. This means that the polynomial $Q$ has degree 4 and all its roots $b_{i}$ are distinct for the generic value $u$. In this section we consider degenerations. It is easy to see that the degree of the polynomial $Q$ can be fixed by 4 with the help of transformations (3.20). It turns out that in all cases the result can be parameterized by a pair of solutions of some degenerations of the hypergeometric equation.

Degeneration 1. Suppose $Q=a\left(x-b_{1}\right)^{2}\left(x-b_{2}\right)\left(x-b_{3}\right)$; then

$$
\frac{R}{Q}=\frac{k_{1}}{x-b_{1}}+\frac{f_{1}}{\left(x-b_{1}\right)^{2}}+\frac{k_{2}}{x-b_{2}}+\frac{k_{3}}{x-b_{3}},
$$

where $k_{1}, k_{2}, k_{3}$ are constants such that $k_{1}+k_{2}+k_{3}=3$, and

$$
\begin{gathered}
b_{1}^{\prime}=-a\left(b_{1}-b_{2}\right)\left(b_{1}-b_{3}\right) f_{1}, \quad b_{2}^{\prime}=a\left(b_{2}-b_{1}\right)^{2}\left(b_{2}-b_{3}\right)\left(1-k_{2}\right), \\
b_{3}^{\prime}=a\left(b_{3}-b_{1}\right)^{2}\left(b_{3}-b_{2}\right)\left(1-k_{3}\right), \quad f_{1}^{\prime}=a f_{1}\left(\left(b_{1}-b_{2}\right)\left(b_{1}-b_{3}\right)\left(2-k_{1}\right)+\left(b_{2}+b_{3}-2 b_{1}\right) f_{1}\right) .
\end{gathered}
$$

A general solution of system (5.32) can be parameterized in the following way:

$$
b_{2}=\frac{y_{1}}{y_{2}}, \quad b_{3}=\frac{z_{1}}{z_{2}}, \quad b_{1}=\frac{y_{1}+u z_{1}}{y_{2}+u z_{2}}
$$


where $\left(y_{1}, z_{1}\right),\left(y_{2}, z_{2}\right)$ are two arbitrary solutions of the linear system

$$
y^{\prime}=\left(-\frac{1}{2}+\frac{3 k_{2}+k_{1}-4}{2\left(k_{2}-1\right) u}\right) y+z, \quad z^{\prime}=\frac{k_{1}+k_{2}-2}{\left(1-k_{2}\right) u^{2}} y+\left(-\frac{1}{2}-\frac{3 k_{2}+k_{1}-4}{2\left(k_{2}-1\right) u}\right) z .
$$

The functions $a, f_{1}$ are determined by system (5.32). Note that system (5.33) is equivalent to the Bessel equation

$$
y^{\prime \prime}+y^{\prime}+\left(\frac{1}{4}+\frac{\left(k_{2}-k_{1}\right)\left(k_{1}+k_{2}-2\right)}{4\left(k_{2}-1\right)^{2} u^{2}}\right) y=0 .
$$

Degeneration 2. Suppose $Q=a\left(x-b_{1}\right)^{2}\left(x-b_{2}\right)^{2}$; then

$$
\frac{R}{Q}=\frac{k_{1}}{x-b_{1}}+\frac{f_{1}}{\left(x-b_{1}\right)^{2}}+\frac{k_{2}}{x-b_{2}}+\frac{f_{2}}{\left(x-b_{2}\right)^{2}},
$$

where $k_{1}, k_{2}$ are constants such that $k_{1}+k_{2}=3$, and

$$
\begin{array}{cl}
b_{1}^{\prime}=-a\left(b_{1}-b_{2}\right)^{2} f_{1}, & b_{2}^{\prime}=-a\left(b_{1}-b_{2}\right)^{2} f_{2}, \\
f_{1}^{\prime}=a\left(b_{2}-b_{1}\right) f_{1}\left(\left(b_{2}-b_{1}\right)\left(k_{2}-1\right)+2 f_{1}\right), & f_{2}^{\prime}=a\left(b_{1}-b_{2}\right) f_{2}\left(\left(b_{1}-b_{2}\right)\left(k_{1}-1\right)+2 f_{2}\right) .
\end{array}
$$

The general solution is given by

$$
b_{1}=\frac{y_{1}}{y_{2}}, \quad b_{2}=\frac{z_{1}}{z_{2}}
$$

where $\left(y_{1}, z_{1}\right),\left(y_{2}, z_{2}\right)$ are two arbitrary solutions of the linear system

$$
y^{\prime}=\frac{3 k_{1}-5}{2 u} y+u z, \quad z^{\prime}=-\frac{1}{3} y+\frac{3\left(1-k_{1}\right)}{2 u} z .
$$

Notice that the function $y(u)$ satisfies the following second order equation:

$$
y^{\prime \prime}+\left(\frac{u}{3}-\frac{3\left(3 k_{1}-5\right)\left(3 k_{1}-7\right)}{u^{2}}\right) y=0
$$

Degeneration 3. Suppose $Q=a\left(x-b_{1}\right)^{3}\left(x-b_{2}\right)$; then

$$
\frac{R}{Q}=\frac{k_{1}}{x-b_{1}}+\frac{f_{1}}{\left(x-b_{1}\right)^{2}}+\frac{f_{2}}{\left(x-b_{1}\right)^{3}}+\frac{k_{2}}{x-b_{2}}
$$

where $k_{1}, k_{2}$ are constants, such that $k_{1}+k_{2}=3$, and

$$
\begin{gathered}
b_{1}^{\prime}=a\left(b_{2}-b_{1}\right) f_{2}, \quad b_{2}^{\prime}=a\left(b_{2}-b_{1}\right)^{3}\left(1-k_{2}\right), \\
f_{1}^{\prime}=a\left(\left(b_{2}-b_{1}\right)\left(f_{1}^{2}-k_{2} f_{2}\right)-2 f_{1} f_{2}\right), \quad f_{2}^{\prime}=2 a f_{2}\left(\left(b_{2}-b_{1}\right) f_{1}-f_{2}\right) .
\end{gathered}
$$

The general solution can be written in the following form

$$
b_{1}=\frac{y_{1}}{y_{2}}, \quad b_{2}=\frac{y_{1}^{\prime}+u y_{1}}{y_{2}^{\prime}+u y_{2}}
$$


where $y_{1}, y_{2}$ are arbitrary solutions of the Weber equation $y^{\prime \prime}=\left(u^{2}+\left(1-2 k_{2}\right)\right) y$. The functions $a, f_{1}, f_{2}$ are completely determined by the above system.

Degeneration 4. Suppose $Q=a(x-b)^{4}$; then

$$
\frac{R}{Q}=\frac{k_{1}}{x-b}+\frac{f_{1}}{(x-b)^{2}}+\frac{f_{2}}{(x-b)^{3}}+\frac{f_{3}}{(x-b)^{4}},
$$

where $k_{1}=3$, and

$$
b^{\prime}=-a f_{3}, \quad f_{3}^{\prime}=-3 a f_{2} f_{3}, \quad f_{2}^{\prime}=-2 a\left(f_{2}^{2}+f_{1} f_{3}\right), \quad f_{1}^{\prime}=a\left(f_{3}-2 f_{1} f_{2}\right) .
$$

Eliminating $f_{3}, f_{2}, f_{1}$ from this system and choosing $a=-1 / b^{\prime 2}$, one obtains the equation

$$
\frac{b^{\prime \prime \prime}}{b^{\prime}}-\frac{3}{2} \frac{b^{\prime \prime 2}}{b^{\prime 2}}=-2 u \text {. }
$$

Its general solution can be written in the form $b=y_{1} / y_{2}$, where $y_{1}, y_{2}$ are arbitrary solutions of the Airy equation $y^{\prime \prime}=u y$.

Deeper degenerations can be obtained by the restriction that the polynomial $Q$ possesses one or several constant roots. In this case it is convenient to make one of these roots the infinity using transformation (3.20). Consider, for instance, Degeneration 4 under assumption $b=$ const. Choosing the normalization $a=1$, one obtains $f_{3}=0$ and

$$
f_{2}^{\prime}=-2 f_{2}^{2}, \quad f_{1}^{\prime}=-2 f_{1} f_{2} .
$$

The simplest solution $f_{2}=f_{1}=0$ of this system corresponds (for $b=\infty$ ) to the Benney pseudo-potential from Example 1. The solution $f_{2}=0, f_{1} \neq 0$ implies $\psi(u, p)=u p+p \log (p)$, which coincides with Case 4 from Section 3 for $c_{1}=c_{2}=0, c_{3}=1$. Finally, if $f_{2} \neq 0$, one can obtain (up to the equivalence)

$$
\psi_{u} \psi_{p p}=1, \quad \psi_{p p}=\lambda \sqrt{u} \exp \left(-\frac{1}{4 u} \psi_{p}^{2}\right)
$$

In this case the solution cannot be expressed in terms of elementary functions.

For general system (4.28) the fact that some roots $b_{i}$ are constant, is equivalent to the equality $k_{i}=1$ for corresponding values $k_{i}$. Solutions of system (4.28) for such degenerations can be extracted from (4.30), (4.31). We omit the explicit formulae for such cases and the analysis of the case of constant $b_{i}$ for Degenerations 1-3.

Let us describe particular solutions from Section 3 in the context of Sections 4, 5. It turns out that Case 5 with $c_{1}^{2} \neq 8 c_{2}$ is equivalent to (4.28), where $b_{1}=-b_{2}, b_{3}=-b_{4}, k_{2}=k_{1}$ $k_{4}=k_{3}$. Namely,

$$
k_{1}=\frac{3 c_{3}-c_{1}}{4 c_{3}}, \quad k_{3}=\frac{3 c_{3}+c_{1}}{4 c_{3}} .
$$

for $c_{2}=c_{1}^{2} / 8-c_{3}^{2} / 8$. 
Case 6 with $c_{1}^{2} \neq 4 c_{2}$ is equivalent to (4.28), where two roots $b_{i}$ are constant. The right hand side of (4.25) reduces to the form

$$
\frac{1}{x}+\frac{k_{1}}{x-b_{1}}+\frac{k_{2}}{x-b_{2}}
$$

where $x=\psi_{p}$,

$$
k_{1}=\frac{c_{3}-c_{1}}{2 c_{3}}, \quad k_{2}=\frac{c_{3}+c_{1}}{2 c_{3}} .
$$

for $c_{2}=c_{1}^{2} / 4-c_{3}^{2} / 4$.

In Case 7 with $c_{1}^{2} \neq-4 c_{2}$ we have $Q(x)=a x\left(x-b_{1}\right)\left(x-b_{2}\right)$ and

$$
\frac{R}{Q}=\frac{k_{1}}{x-b_{1}}+\frac{k_{2}}{x-b_{2}}
$$

where

$$
k_{1}=\frac{3 c_{3}-c_{1}}{2 c_{3}}, \quad k_{2}=\frac{3 c_{3}+c_{1}}{2 c_{3}}
$$

for $c_{2}=-c_{1}^{2} / 4+c_{3}^{2} / 4$. Notice that in this case $k_{1}+k_{2}=3$. It is easy to verify that if the right hand side of (4.25) has the form (5.35), then the constants $k_{1}, k_{2}$ can be arbitrary, and $b_{1}, b_{2}$ satisfy the system

$$
b_{1}^{\prime}=a\left(b_{1}-b_{2}\right)\left(1-k_{1}\right), \quad b_{2}^{\prime}=a\left(b_{2}-b_{1}\right)\left(1-k_{2}\right)
$$

In the case, when $k_{1}+k_{2}=2$, system (5.36) possesses the solution $a=1 ; b_{i}=u+t_{i}$, where $k_{1}=1+1 /\left(t_{1}-t_{2}\right), k_{2}=1+1 /\left(t_{2}-t_{1}\right)$. It corresponds to Case 4 from Section 3 with $c_{1} \neq 0, c_{2}^{2} \neq 4 c_{1} c_{3}$. For the Kupershmidt chain (see Example 2),

$$
\frac{R}{Q}=\frac{k_{1}}{x-b_{1}},
$$

$a=1$ and $b_{1}=u+t_{1}$.

For Case 2 with $c_{1} \neq 0, c_{2}^{2} \neq 4 c_{1} c_{3}$ we have (5.35), (5.36) and $k_{1}+k_{2}=1$. Under the latter condition, (5.36) possesses the solution $a=1 / u ; b_{i}=t_{i} u$, where $k_{1}=t_{2} /\left(t_{2}-t_{1}\right), k_{2}=$ $t_{1} /\left(t_{1}-t_{2}\right)$.

Case 1 corresponds to a constant solution of system (5.36), which exists for $k_{1}=k_{2}=1$. It was already mentioned in Section 3 that Case 3 is equivalent to Case 1.

\section{Conclusion}

We apply the method of hydrodynamic reductions to classify integrable Vlasov-type equations of the form (1.1), (1.2). In this paper the simplest case of one function $U(x, t)$ is completely 
analyzed. In the next paper we are going to solve a more complicated problem of classification of integrable Vlasov-type equations in the case of two functions $U_{1}(x, t), U_{2}(x, t)$. It turns out that there exist several essentially different classes of integrable functions $\psi\left(U_{1}, U_{2}, p\right)$. One of such classes corresponds to two-component $(2+1)$-dimensional hydrodynamic-type systems. This class was constructed in the paper [14]. Note that examples of integrable functions $\psi\left(U_{1}, \ldots, U_{n}, p\right)$ appeared earlier in other papers, where other approaches were used. In particular, functions $\psi$, associated with algebraic curves of an arbitrary genus, were constructed in [15]. An integrable function $\psi\left(U_{1}, \ldots, U_{n}, p\right)$ was constructed from any $n$-dimensional Frobenius manifold in [16].

As it was shown in [10], equation (2.12) in the case of the Benney chain (see Example 1) is nothing but the Loewner equation well known in the theory of conformal mappings. The results obtained in our paper can be of interest in connection with the so-called Laplacian growth problem (see [17] and references therein). Moreover, each integrable case leads to an integrable hydrodynamic chain similar to the Benney chain (see Example 1.) If the range of the discrete variable $k$ is the set of all integers, the corresponding hydrodynamic chains can be constructed rather easily. However, the problem of a "right" truncation of such chains to the set of non-negative values of $k$ is not trivial. We are going to write a separate paper on the subject.

Acknowledgments. Authors thank B.A. Dubrovin and E.V. Ferapontov for fruitful discussions. We are grateful to the ESF Research Network MISGAM for partial financial support of the ISLAND-3 conference, where the work on this paper was initiated. V.S. thanks IHES and A.O. thanks MPIM for hospitality and financial support. M.P. was partially supported by the Russian-Italian Research Project 06-01-92053. V.S. was partially supported by the RFBR grants 08-01-461 and NS 1716.2003.1.

\section{References}

[1] V.E. Zakharov, Benney's equations and quasi-classical approximation in the inverse problem method, Funct. Anal. Appl., 14 No. 2 (1980) 89-98. V.E. Zakharov, On the Benney's Equations, Physica 3D (1981) 193-200.

[2] M.V. Pavlov, Classification of integrable hydrodynamic chains and generating functions of conservation laws, J. Phys. A: Math. Gen. 39(34) (2006) 10803-10819.

[3] M.V. Pavlov, Algebro-geometric approach in the theory of integrable hydrodynamic-type systems. Comm. Math. Phys., 272(2) (2007) 469-505.

[4] D.J. Benney, Some properties of long nonlinear waves, Stud. Appl. Math. 52 (1973) 45-50.

[5] J. Gibbons, Collisionless Boltzmann equations and integrable moment equations, Physica 3D (1981) 503-511. 
[6] B.A. Kupershmidt, Yu.I. Manin, Long wave equation with free boundaries. I. Conservation laws. Func. Anal. Appl., 11 No. 3 (1977) 188-197. B.A. Kupershmidt, Yu.I. Manin, Long wave equations with a free surface. II. The Hamiltonian structure and the higher equations, Func. Anal. Appl., 12 No. 1 (1978) 25-37. D.R. Lebedev, Yu.I. Manin, Conservation laws and representation of Benney's long wave equations, Phys. Lett. A, 74 No. 3,4 (1979) 154 156. D.R. Lebedev, Benney's long wave equations: Hamiltonian formalism, Lett. Math. Phys., 3 (1979) 481-488.

[7] B.A. Kupershmidt, Deformations of integrable systems, Proc. Roy. Irish Acad. Sect. A, 83(1) (1983) 45-74. B.A. Kupershmidt, Normal and universal forms in integrable hydrodynamical systems, Proceedings of the Berkeley-Ames conference on nonlinear problems in control and fluid dynamics (Berkeley, Calif., 1983), in Lie Groups: Hist., Frontiers and Appl. Ser. B: Systems Inform. Control, II, Math Sci Press, Brookline, MA, (1984) 357-378.

[8] M.V. Pavlov, The Kupershmidt hydrodynamic chains and lattices, IMRN, (2006) 1-43 (Article ID 46987).

[9] E.V. Ferapontov, K.R. Khusnutdinova, On integrability of (2+1)-dimensional quasilinear systems, Comm. Math. Phys. 248 (2004) 187-206, E. V. Ferapontov, K.R. Khusnutdinova, The characterization of 2-component $(2+1)$-dimensional integrable systems of hydrodynamic type, J. Phys. A: Math. Gen. 37(8) (2004) 2949 - 2963.

[10] J. Gibbons, S.P. Tsarev, Reductions of Benney's equations, Phys. Lett. A, 211 (1996) 19-24. J. Gibbons, S.P. Tsarev, Conformal maps and reductions of the Benney equations, Phys. Lett. A, 258 (1999) 263-270.

[11] S.P. Tsarev, On Poisson brackets and one-dimensional Hamiltonian systems of hydrodynamic type, Soviet Math. Dokl., 31 (1985) 488-491. S.P. Tsarev, The geometry of Hamiltonian systems of hydrodynamic type. The generalized hodograph method, Math. USSR Izvestiya, 37 No. 2 (1991) 397-419. 1048-1068.

[12] M.V. Pavlov, Classification of the Egorov hydrodynamic chains. Theor. Math. Phys. 138 No. 1 (2004) 55-71.

[13] A. Odesskii and V.Sokolov, On (2+1)-dimensional hydrodynamic-type systems possessing pseudopotential with movable singularities, to appear in Func. Anal. Appl.

[14] A.V. Odesskii, A family of (2+1)-dimensional hydrodynamic-type systems possessing pseudopotential, arXiv:0704.3577v3 [math. AP], to appear in Selecta Mathematica.

[15] I.M. Krichever, The $\tau$-function of the universal Whitham hierarchy, matrix models and topological field theories, Comm. Pure Appl. Math., 47 (1994), no. 4, 437-475.

[16] B.A. Dubrovin, Geometry of 2D topological field theories. In Integrable Systems and Quantum Groups, Lecture Notes in Math. 1620 (1996), 120-348. 
[17] A.V. Zabrodin, The dispersionless limit of the Hirota equations in some problems of complex analysis. Theoret. and Math. Phys., 129 (2), 1511-1525, 2001. 